\title{
Comunicação
}

[Communication]

\section{Aspectos radiográficos de condrodistrofia felina}

\author{
[Radiographic aspects of feline chondrodystrophy] \\ M.A.R. Feliciano ${ }^{1}$, C.A.L. Leite $^{2}$, W.R.R. Vicente ${ }^{3}$, T. Silveira ${ }^{4}$ \\ ${ }^{1}$ Aluno de pós-graduação - FCAV-UNESP - Jaboticabal, SP \\ ${ }^{2}$ Departamento de Veterinária - UFLA - Lavras, MG \\ ${ }^{3}$ Faculdade de Ciências Agrárias e Veterinárias - UNESP - Jaboticabal, SP \\ ${ }^{4}$ Médica veterinária autônoma - Lavras, MG
}

Segundo Burk e Ackerman (1996), as afecções osteoarticulares congênitas, hereditárias e do desenvolvimento são comuns na rotina clínica e radiológica em cães, porém são consideradas incomuns em felinos. Para Jesyk (1985), a maioria das anomalias conformacionais é oriunda de interações genéticas autossômicas dominantes com expressividade bastante variável.

Como citado por Johnson e Watson (2004), o crescimento ósseo ocorre principalmente por ossificação endocondral, porém, de forma secundária, há também crescimento aposicional. Dessa forma, a condrodistrofia é uma condição na qual a cartilagem epifisária dos ossos longos ossifica prematuramente e, consequentemente, os membros param o crescimento antes da maturidade (Chia-Hung et al., 2007). Este abrandamento do crescimento afeta a ulna antes do rádio e esta falta de sincronização resulta no encurvamento da articulação do cotovelo. Além dos membros encurtados, as raças condrodistróficas, frequentemente, apresentam o canal vertebral mais estreito. Pedersen et al. (2004) descreveram também que a estrutura das superfícies articulares varia bastante em animais condrodistróficos. Esta afecção óssea predispõe à doença articular degenerativa. Estudos, em 1964, postularam que a curvatura dos membros é proporcional ao grau de condrodistrofia e está relacionada à ocorrência de calcificação de discos intervertebrais (Lappalainen et al., 2001).

Recebido em 10 de março de 2008

Aceito em 4 de fevereiro de 2009

E-mail: marcusfeliciano@yahoo.com.br
Em cães, é comum em raças como Basset Hound, Pequinês, Pug e Teckel, e altamente indesejável no Malamute do Alasca e no Pastor Alemão (Pedersen et al., 2004), não havendo diferenças significativas entre os machos e as fêmeas na ocorrência de condrodistrofia (Lamoreux, 1942). Como é incomum nos gatos, não há relatos de raças mais acometidas.

O diagnóstico dessas afecções é importante para o controle dos cruzamentos, seja por parte de veterinários ou de criadores. Essas alterações, geralmente, consistem em achados radiográficos ocasionais ou deformidades e anomalias sem demais consequências clínicas para o animal (Burk e Ackerman, 1996). De acordo com Schwarz (2003), o único sinal clínico evidente é a claudicação persistente ou intermitente. O exame físico e a observação do tamanho, conformação e locomoção são complementados pelos exames de imagem e também por análises séricas e histopatológicas. Os métodos de diagnóstico por imagem, além de não invasivos, permitem a avaliação do esqueleto sem afetar o crescimento e o desenvolvimento (Brawner, 1998).

A condrodistrofia, ao exame radiográfico, caracteriza-se por deformidades angulares osteoarticulares, podendo ocorrer também encurtamento do membro devido à hipoplasia dos ossos (Johnson e Watson, 2004). O objetivo deste trabalho foi relatar os aspectos radiográficos de um caso de condrodistrofia felina, alteração osteoarticular incomum nesta espécie. 
Uma gata, sem raça definida, foi levada ao hospital veterinário, para realização de ovariohisterectomia eletiva. Ao exame físico, verificouse que o animal apresentava deformidade em todos os membros, mas não foi notada qualquer sintomatologia clínica que comprometesse a vida do animal.
Por meio do exame radiográfico dos membros anteriores e posteriores, nas projeções mediolateral e craniocaudal, visualizou-se alteração na angulação óssea de úmero, rádio, ulna, carpo, metacarpo, fêmur, tíbia, fíbula, tarso e metatarso. Pôde-se visualizar também um alargamento das articulações relacionadas às estruturas ósseas dos membros (Fig. 1 e 2).

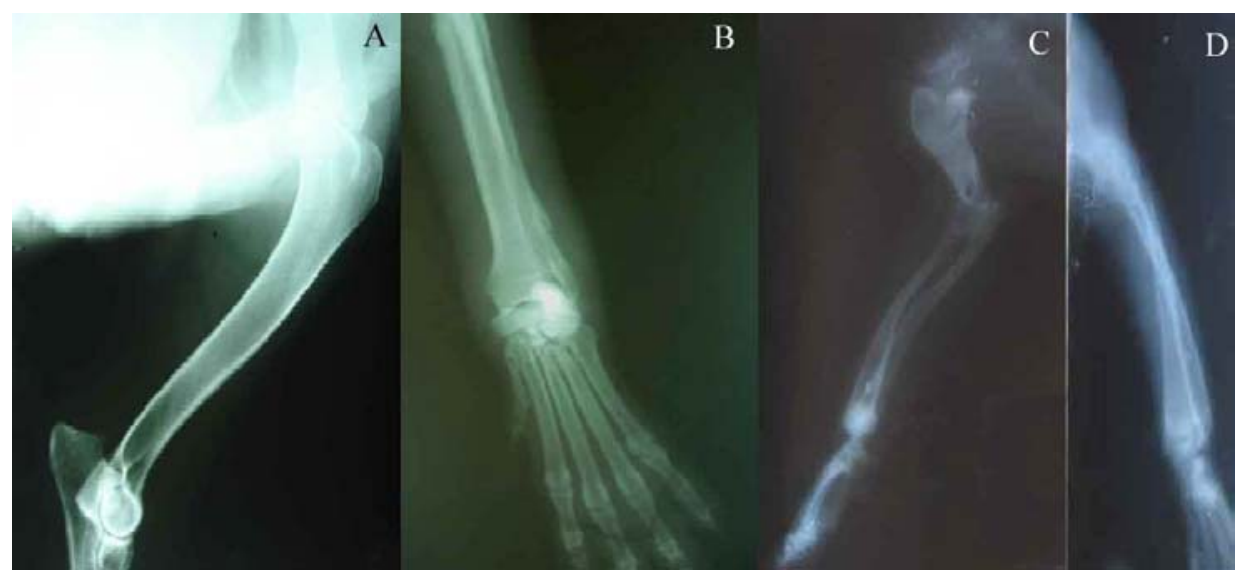

Figura 1. Imagens radiográficas de membros torácicos e articulações de gato. A e B: sem alterações; C e D: encurvamento ósseo e espessamento da região articular.

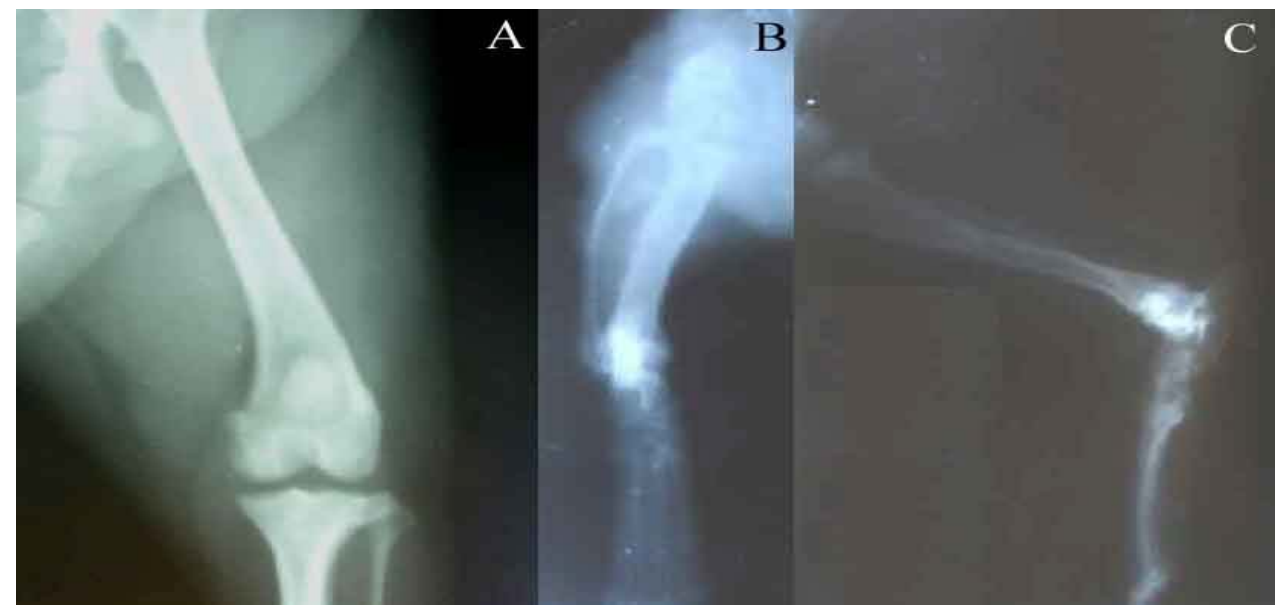

Figura 2. Imagens radiográficas de membros pelvinos e articulações de gato. A: sem alterações; B e C: encurvamento ósseo e espessamento da região articular.

Johnson e Watson (2004) e Pedersen et al. (2004) descrevem que os sinais radiográficos que caracterizam a condrodistrofia são as deformidades angulares das articulações, em suas diversas apresentações. Também é possível observar ossos menores e malformados em relação ao membro contralateral. Dessa forma, em analogia à espécie canina, foi possível observar alterações compatíveis com condrodistrofia, o que sugere a ocorrência desta em felinos.

Concluiu-se que, mesmo sendo incomum em felinos, a condrodistrofia pode ser um achado radiográfico incidental, mas de grande importância para o aspecto epidemiológico da doença.

Palavras-chave: gato, condrodistrofia, radiologia 


\begin{abstract}
Congenital, hereditary, and developmental diseases are common in small animals radiology. Chondrodystrophy is characterized to osteoarticular angular deformities. The aim of this study is to relate a case of feline chondrodystrophy, alteration uncommon in this species. The animal presented bone alterations in limbs, without healthy commitment at clinical examination. At radiographic evaluation, bone angulation was altered in long bones with articulation enlargement. It was concluded that, even uncommon in felines, chondrodystrophy may be an incidental radiographic finding, but with importance to epidemiologic aspect of this disease.
\end{abstract}

Keywords: cat, chondrodystrophy, radiology

\section{REFERÊNCIAS BIBLIOGRÁFICAS}

BRAWNER JR., W.R. Diagnostic imaging of skeletal growth and disorders. In: CANINE SKELETAL DEVELOPMENT \& SOUNDNESS: THE NORTH AMERICAN VETERINARY CONFERENCE, 1998, Orlando, FL. Evanston: MultiMédia/Veterinary Learning Systems, 1998. p.19-24.

BURK, R.L.; ACKERMAN, N. The appendicular skeleton. In:_. Small animal radiology and ultrasonography: a diagnostic atlas and text. 2.ed. Philadelphia: W.B. Saunders, 1996. p.427529.

CHIA-HUNG, T.; WEI-CHIH, L.; TSUNGHSIEN, C. et al.Pet parasites infesting the human colon. Gastrointest. Endosc., v.67, p.159-160, 2007.

JESYK, P.K. Constitutional disorders of the skeleton in dogs and cats. In: NEWTON, C.D.; NUNAMAKER, D.M. (Eds). Textbook of small animal orthopaedics. New York: Ivis, 1985. cap. 57.

JOHNSON, K.A.; WATSON, A.D.J. Doenças esqueléticas. In: ETTINGER, S.J.; FELDMAN, E.C. (Eds). Tratado de medicina interna veterinária: doenças do cão e do gato. 5.ed. Rio de Janeiro: Guanabara-Koogan, 2004. p.19882019.
LAMOREUX, W.F. Hereditary chondrodystrophy in the fowl. J. Hered., v.33, p.275-283, 1942.

LAPPALAINEN A.; NORRGARD, M.; ALM, $\mathrm{K}$. et al. Calcification of the intervertebral discs and curvature of the radius and ulna: a radiographic survey offinnish miniature dachshunds. Acta Vet. Scand., v.42, p.229-236, 2001.

PEDERSEN, N.C.; MORGAN, J.P.; VASSEUR, P.B. Doenças articulares de cães e gatos. In: ETTINGER, S.J.; FELDMAN, E.C. (Eds). Tratado de medicina interna veterinária: doenças do cão e do gato. 5.ed. Rio de Janeiro: Guanabara-Koogan, 2004. p.1962-1988.

SCHWARZ, P.D. Osteocondrose. In: TILLEY, L.P.; SMITH Jr., F.W.K. (Eds). Consulta veterinária em 5 minutos: espécies canina $e$ felina. 2.ed. Barueri: Manole, 2003. p.1022-1023. 